\title{
MIMO-OFDM Systems Performance Enhancement Based Peaks Detection Algorithm
}

\author{
http://dx.doi.org/10.3991/ijim.v7i3.2302 \\ Omar Daoud $^{1}$, Qadri Hamarsheh ${ }^{1}$ and Wael Al-Sawalmeh ${ }^{2}$ \\ ${ }^{1}$ Philadelphia University, Amman, Jordan \\ ${ }^{2}$ Al-Hussin Bin Talal University, Ma’an, Jordan
}

\begin{abstract}
-this work proposes a new algorithm to enhance the performance of the speaker verification over the communication systems based Multiple-Input Multiple-Output (MIMO) - Orthogonal Frequency Division Multiplexing (OFDM) techniques. The algorithm mainly tackles and overcomes the effect of the high Peak-to-Average Power Ratio problem that is found in OFDM systems, MIMOOFDM combination has been developed to meet the rapidly increment in the users demand such as the ubiquitous transmission, imposing new multimedia applications and wireless services. In this algorithm, wavelets techniques have been used to denoise the affected OFDM symbol by high PAPR values. After that and based on adaptive threshold method the local maxima and minima will be determined and replaced by the average of them and their surrounding neighbors; Denoise OFDM and Replace PAPR (DORP).
\end{abstract}

A system performance investigation process will be accomplished based on both of numerical method and MATLAB simulation. Moreover, a comparison has been made to check the validity of our proposition either with our previously published work or with the literature. Although, the achieved results show that the proposed work has lower PAPR values; an additional complexity has been added to transceiver's structure. Moreover, and as a result to the comparison with the conventional systems, the bit error rate (BER) performance has been improved for the same bandwidth occupancy.

As a validity process a comparison has been made with the current values found in the literature and we have achieved around $30 \%$ PAPR extra reduction. That is in addition to around $90 \%$ verification rate and noise immunity.

Index Terms-MIMO, OFDM, Peak-to-Average Power Ratio, wavelet, Eigen vectors component.

\section{INTRODUCTION}

Over the last decade and due to the overwhelming huge data that the users' create, transmit and/or manage; a challenge has been made to the wireless communication systems vendors to ease such complexities. Moreover, the main challenge of the new generation of wireless cellular systems is the reliability of providing data rate of around $100 \mathrm{Mbps}$ and $30 \mathrm{Mbps}$ for the downlink and uplink physical layer transmission, respectively. Therefore, researchers have turned their attentions towards the combination of two powerful techniques, namely Orthogonal Frequency Division Multiplex (OFDM) and Multiple-Input Multiple-Output (MIMO) technology. This will produce ad- vanced wireless techniques with high capacity and restrain the growth in the multimedia applications, internet access either wired or wireless and other needed applications in the new generation mobile systems. One of the main challenging issues is Speaker verification. It has been the topic of active research for many years, and has many important applications where propriety of information is a concern [1]. This is revealing that the speaker verification system is a difficult task. Typically, these intricacies contain the needed amount of speech for accurate verification and estimation; and the threshold estimation for acceptance and rejection [1-3]. An example of the technologies that have been developed to meet the increasing demand of reliability, coverage, the new services is the combination between two main techniques in cellular systems; MIMOOFDM. This is to improve the system reliability and performance.

Orthogonal frequency division multiplexing has emerged as an efficient multicarrier modulation scheme for wireless, frequency selective, communication channels. Ease of implementation, high spectral efficiency, resilience to impulse noise and multipath are a few advantages of OFDM systems. It is a multi-carrier system which utilizes a parallel processing technique allowing the simultaneous transmission of data on many closely spaced, orthogonal sub-carriers. Inverse fast Fourier transforms (IFFT) and fast Fourier transform in a conventional OFDM system are used to multiplex the signals together and decode the signal at the receiver respectively. The system adds cyclic prefixes (CP) before transmitting the signal. The purpose of this is to increase the delay spread of the channel so that it becomes longer than the channel impulse response. The purpose of this is to minimize inter-symbol interference (ISI). However, the CP has the disadvantage of reducing the spectral containment of the channels. The main challenge of the new generation of wireless cellular systems is the reliability of providing data rate of around [4]. However, the peak-to-average power ratio (PAPR) is appears as a major drawback in the OFDM signal. This deficiency could be defined as a large envelope fluctuation. As a consequence, it limits the efficiency of the non-linear devices such as the power amplifiers, mixers, and analog to digital converters. Then they will operate at lower average power. As a result of this drawback, the signals envelop will be a Raleigh distributed in addition to a complex Gaussian process behavior. Various schemes have been developed to reduce high PAPR and classified as distortion and distortion-less methods in OFDM signals [5-9]. Those techniques reduce the PAPR at the expense of an increased, irreducible, symbol error rate at the receiver in addition to transmitting 
side information resulting in a reduced throughput. Previously in another publication [10], a new proposition of PAPR reduction technique to overcome those complexities shows that the average power is equal to the number of modulated OFDM subcarriers as shown in the mathematical derivation. Starting from the definition of the PAPR as

PAPR $=10 \log _{10}\left\{\frac{P_{\text {peak }}}{P_{\text {avg }}}\right\}$

where, $P_{\text {peak }}$ is the maximum power of an OFDM symbol, and $P_{\text {avg }}$ is the average power. The PAPR can be reformulated as:

$$
\text { PAPR }=\frac{|x(t)|^{2}}{\frac{1}{N T} \int_{0}^{N T}|x(t)|^{2} d t}
$$

where $T$ is the symbol duration, $x(t)$ is the OFDM symbol at time, $t$, which can be expressed as $x(t)=\frac{1}{\sqrt{N}} \sum_{n=0}^{N-1} X_{n} e^{j 2 \pi f_{0} n t}, 0 \leq \tau \leq N \mathrm{~N} . X_{\mathrm{n}}$ is the data modulating the $n$-th is sub carrier and $f_{0}$ is the nominal subcarrier frequency spacing. Moreover, the average power of the OFDM symbol presented in (2) can be written as:

$P_{\mathrm{avg}}=\sum\left\{\left|x_{i}(t)\right|^{2}\right\}=\frac{1}{T} \int_{0}^{T}|x(t)|^{2} d t$

$P_{\mathrm{avg}}=\frac{1}{T} \int_{0}^{T}\left|\sum_{v=0}^{N-1} c_{v} e^{j 2 \pi \frac{v}{N T} t^{v}}\right|^{2} d t$

$P_{\text {avg }}$

$=\frac{1}{T} \int_{0}^{T}\left(\sum_{v=0}^{N-1} c_{v} \cos \left(2 \pi \frac{v}{N T} t_{v}\right)\right)^{2}+$

$\left(\sum_{v=0}^{N-1} c_{v} \sin \left(2 \pi \frac{v}{N T} t_{v}\right)\right)^{2} d t$

$P_{\mathrm{avg}}=\frac{1}{\mathbf{T}} \int_{0}^{\mathbf{T}}\left(\sum_{\mathbf{v}=0}^{\mathbf{N}-1} \mathbf{c}_{\mathbf{v}}^{2}\right) \mathbf{d t}$

where $c_{v}$ is the magnitude of the modulated data .

For simplicity, if BPSK modulation is used without any channel coding techniques, then $|\boldsymbol{c} \boldsymbol{v}|=1$, when $\tau \in[0, \mathrm{~T}]$. This can be substituted in (13). The result from this substitution leads to a direct relationship between the average power and the total number the IFFT points, $N$, which can be taken directly from
$P_{\mathrm{avg}}=N \frac{1}{\mathbf{T}} \int_{0}^{\mathbf{T}} \mathbf{c}_{\mathbf{v}}^{2} \mathbf{d t}=N$,

From (7) the average power is equal to the number of the BPSK-modulated orthogonal subcarriers. As already mentioned, the maximum peak amplitude is $N$, therefore, the maximum power of the OFDM symbol is $N^{2}$. As a result, from (1), the PAPR of uncoded BPSK will be $10 \log _{10}\left(P_{\mathrm{avg}}\right) \mathrm{dB}$. Therefore, the PAPR will be decreased if the average power of the OFDM symbol is decreased. Based on (7), a proposition of a new technique has been made to overcome the computational complexity that has been found in the literature as shown in Figure 1.

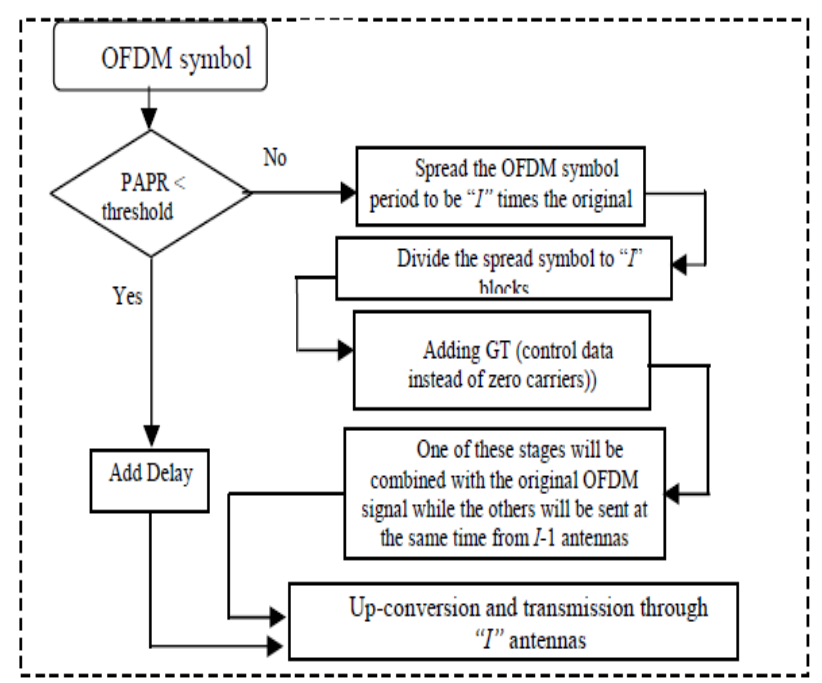

Figure 1. The flowchart of the algorithm [10]

In this paper, a new proposition of reducing the PAPR effect is introduced; by seeking the affected OFDM symbol by PAPR and replace these peaks by the average of the surrounding neighbors in addition to the value of this peak, which gives an enhancement up to $85 \%$ of the values of the complementary cumulative distribution function (CCDF).

The rest of paper is organized as follows; the introduced structure of the MIMO-OFDM system models is defined in Section 2, the numerical and simulation results are presented in Section 3, while the last section summarizes the conclusion.

\section{THE MIMO-OFDM SYSTEM DESCRITION}

In this subsection, a new algorithm has been proposed as shown in Figure 2 (a). Identifying and analyzing the peaks and valleys of OFDM. Signal detection algorithm has been implemented using MATLAB software It can be summarized by the following steps, while the flowchart of this process is shown in Figure 2 (b):

1. Read a segment of the OFDM signal.

2. Denoise the OFDM signal from additive white Gaussian noise (AWGN) using wavelets technique [11]. In this step the unwanted random addition to a wanted signal is removed using the following sub steps

a. Applying discrete wavelet transform DWT to the noisy signal.

b. Applying soft thresholding operator (wavelet shrinkage) [12] to highlight large values of wavelet 
PAPER

MiMO-OFDM Systems Performance EnHANCEMEnt Based PeAKs Detection Algorithm

coefficients which almost correspond to the OFDM signal and suppress small values which correspond to noise.

c. Applying inverse discrete wavelet transform IDWT to the thresholded wavelet coefficients to reconstruct a denoised OFDM signal.

3. Define an adaptive magnitude threshold required for peak detection algorithm using the following formulas [27]:

$$
\mathbf{T}=\frac{\left(\max +a b s_{-} a v g\right)}{2}+(\mathbf{K} * \text { abs_dev })
$$

Where

max is the maximum value in the OFDM signal.

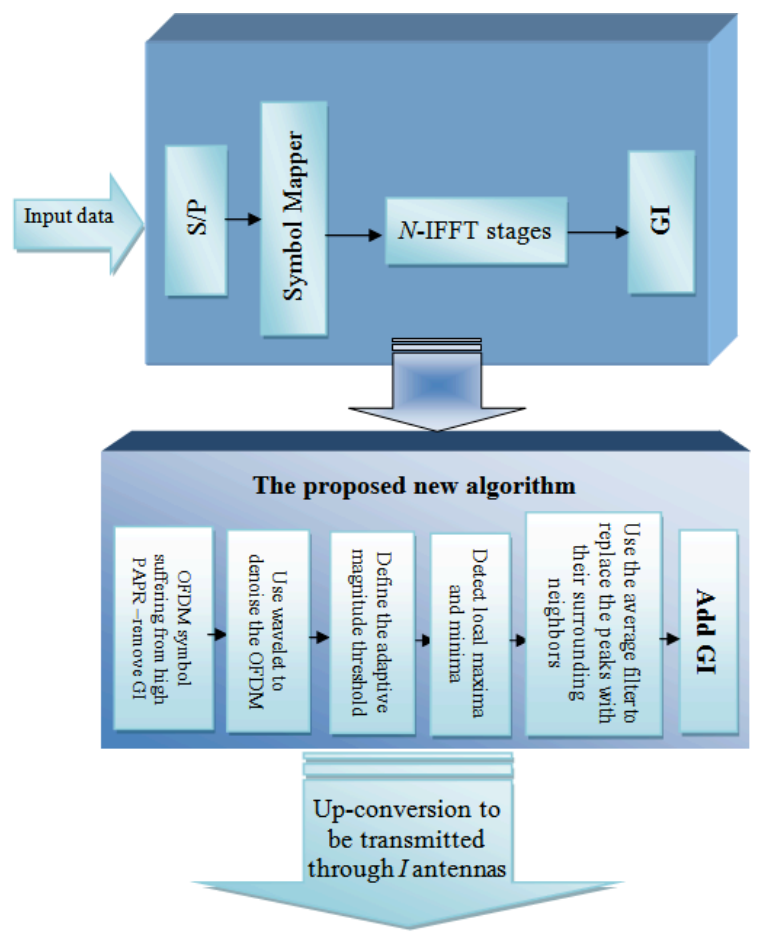

(a)

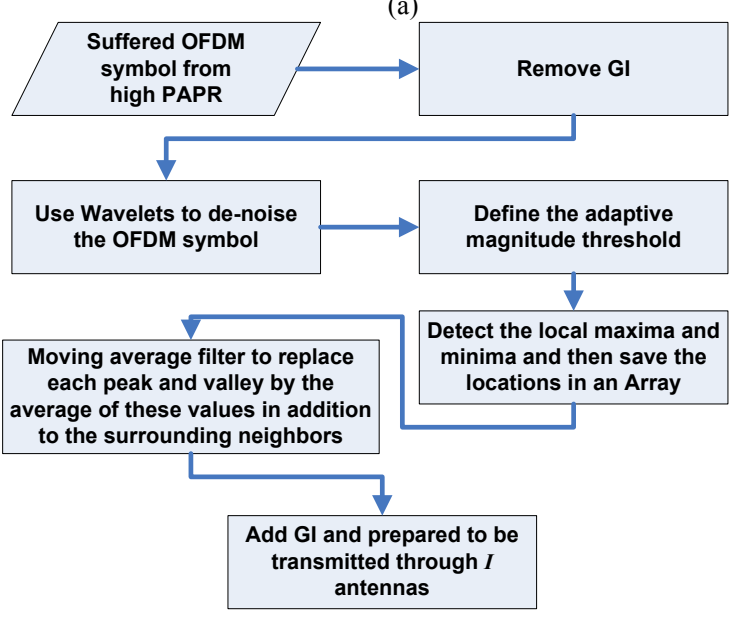

(b)

Figure 2. (a) the block diagram of the proposed work, (b) the flowchart of the proposed algorithm abs_avg is the average of the absolute values in the OFDM signal.

abs_dev is the mean absolute deviation.

$\mathrm{K}$ is a user defined constant $(\mathrm{k}=1)$.

The obtained threshold value is used to determine if each peak (valley) is significantly larger (or smaller) than the data around it.

4. Detect local maxima (peaks) and local minima (valleys), save these locations in an array(values and indices).

5. Use moving average filter for the found vector's points with their neighbors surrounding them in original OFDM signal and save the result into corresponding points of the original signal.
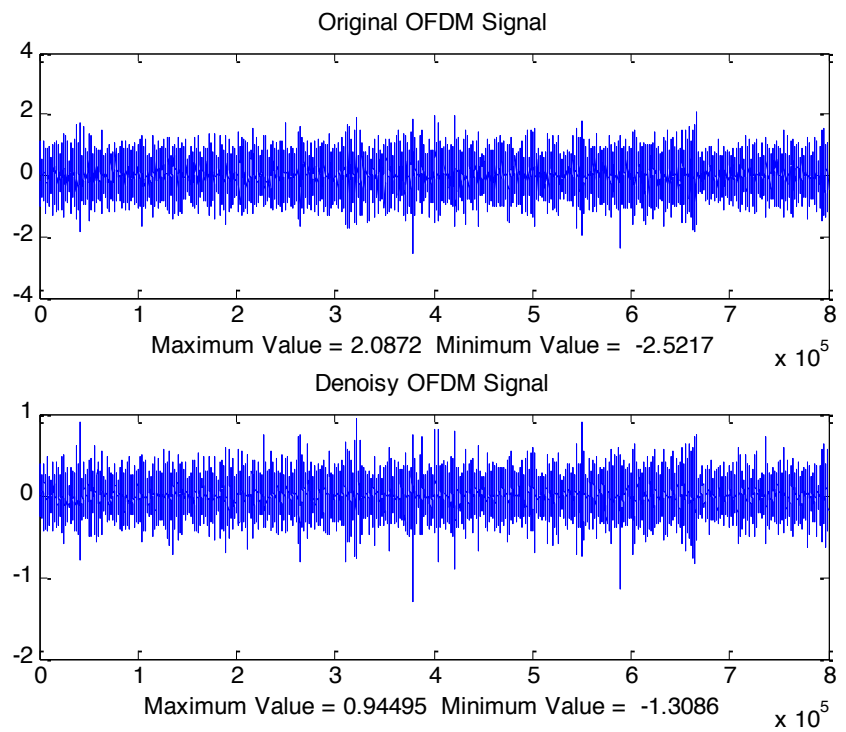

(a)
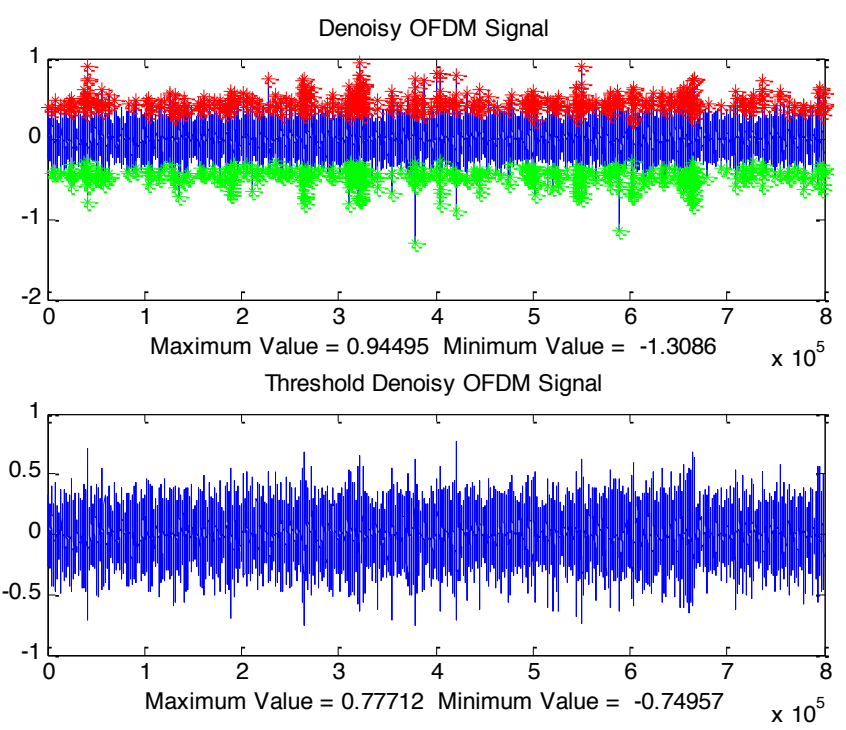

(b)

Figure 3. The results of applying the new algorithm. 3.a The result of denoisy the OFDM signal 3.b the resultant OFDM signal after applying the proposed thresholding technique. 
The results of this procedure are clearly found in Figure 3. Figure $3 \mathrm{a}$ shows the effect of denoisy the original OFDM signal. In this part, it is clearly shown the result of applying some denoisy methods; the maximum value has been reduced from 2.087 to 0.94495 and the minimum value also has been modified to be -1.3086 with a reduction ratio equals to $52 \%$. In Figure $3 \mathrm{~b}$, the results of high peaks and valleys have been modified by imposing the proposed algorithm and its clearly shown that the maximum value has been improved to be 0.77712 with an enhancement ratio equals to almost $18 \%$.

The next section describes the results from the simulation of the proposed work compared with the conventional techniques. The BER will be the key factor in differentiating the proposed work over the conventional ones in the literature, is in addition to the CCDF curves; which is a performance metric independent of the transmitter amplifier. Given the reference level $P A P R_{0}>0$, the probability of a PAPR being higher than the reference value is the CCDF and is expressed as follows [6]:

$$
\mathrm{CCDF}\left(\mathrm{PAPR}_{0}\right)=\operatorname{Pr}\left\{\mathrm{PAPR}>\mathrm{PAPR}_{0}\right\}
$$

For practical reasons, the CCDF of PAPR is calculated based on the percentage of the OFDM/WPM frames for which PAPR exceeds the threshold $P A P R_{0}$. These results will be extracted from a Multipath condensed channel.

\section{SIMULATION RESULTS AND DISCUSSION}

In this section the results of the comparison between the conventional MIMO-OFDM systems with the proposed one has been made. Thus, the conventional OFDM-based FFT transceiver has been simulated using MATLAB and its performance is compared with the one that is based on wavelet packet. We also compare the performance of the proposed system in identical channel conditions with the theoretical representation which is based on randomly generated data.

In order to verify the validity of our analytically derived technique, the MATLAB simulation program was performed with the following factors:

- Tested speech signals were recorded with a spectral frequency of $4 \mathrm{kHz}$ and sampling frequency $16 \mathrm{kHz}$, over about $3 \mathrm{~s}$ time duration. Each speaker recorded 4 times and they are classified as 6 females and 8 males of different ages participated in utterances recording. This is in addition to the theoretical data which is randomly generated to check the system effectiveness.

- different modulation techniques (BPSK and QPSK),

- IFFT size of 512,

Table 1 shows the promising performance of the proposed work; DORP with the ones found in the literature.

Using an intensive modulation technique; QPSK, the MIMO-OFDM CCDF value has been reduced at the $20 \mathrm{~dB}$ threshold around $89 \%$ for the DORP, which equals to $75 \%$ additional reduction over the conventional work that is based on FFT. Furthermore, when the modulation technique has been changed to be BPSK, these reduction ratios have been slightly decreased. For the same threshold value, MIMO-OFDM based FFT has around $77 \%$ reduction improvement over the conventional PAPR reduction techniques.
TABLE I.

A PERFORMANCE COMPARISON BETWEEN THE PROPOSED WORK AND THE USED TECHNIQUES IN THE LITERATURE FOR THE SPREADING RATE OF 0.5

\begin{tabular}{|c|c|c|c|c|c|c|}
\hline \multirow[b]{2}{*}{ 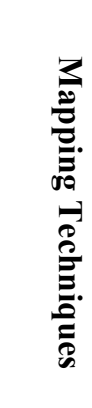 } & & \multicolumn{5}{|c|}{$\begin{array}{c}\text { MIMO-OFDM systems' Performance } \\
\text { P(PAPR }>10(\mathrm{~dB})),(\%)\end{array}$} \\
\hline & 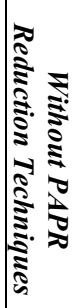 & 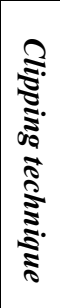 & 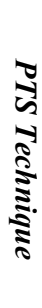 & 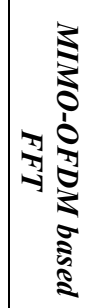 & $\underset{\overbrace{}}{\overbrace{}}$ & $\begin{array}{l}\text { Improvement } \\
\text { percentage of } \\
\text { using DORP } \\
\text { over the } \\
\text { literature }(\%)\end{array}$ \\
\hline $\begin{array}{c}\text { QPS } \\
\mathbf{K}\end{array}$ & $\stackrel{\substack{x \\
\sigma_{1}}}{+}$ & 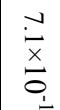 & $\begin{array}{l}\hat{i} \\
x \\
\dot{0}\end{array}$ & $\begin{array}{l}\overrightarrow{0} \\
\dot{0} \\
\dot{x} \\
\dot{0}\end{array}$ & 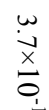 & $46 \%$ \\
\hline BPSK & $\begin{array}{l}\infty \\
\dot{x} \\
\dot{0}\end{array}$ & $\begin{array}{l}\infty \\
\dot{w} \\
\dot{+} \\
\stackrel{x}{0} \\
\dot{1}\end{array}$ & $\stackrel{\infty}{\frac{\infty}{0}}$ & 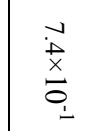 & $\begin{array}{l}u \\
\dot{\alpha} \\
x \\
\dot{0} \\
0\end{array}$ & $32 \%$ \\
\hline
\end{tabular}

Figures 4 and 5 show the simulation part that is based on the CCDF curves. These results check the performance of our system from reducing the PAPR problem point of view for two different modulation techniques; QPSK and BPSK, respectively. These Figures compare the threshold value against the probability that the PAPR will exceed the threshold value. From these Figures the reduction improvements are clearly shown over what have been achieved in the literature for the conventional MIMOOFDM systems.

The CCDF curves in both Figures confirm the reliability of the proposed work for combating the PAPR problem. Thus, the performance of the MIMO-OFDM based FFT is still better than that of the conventionalPAPR re-

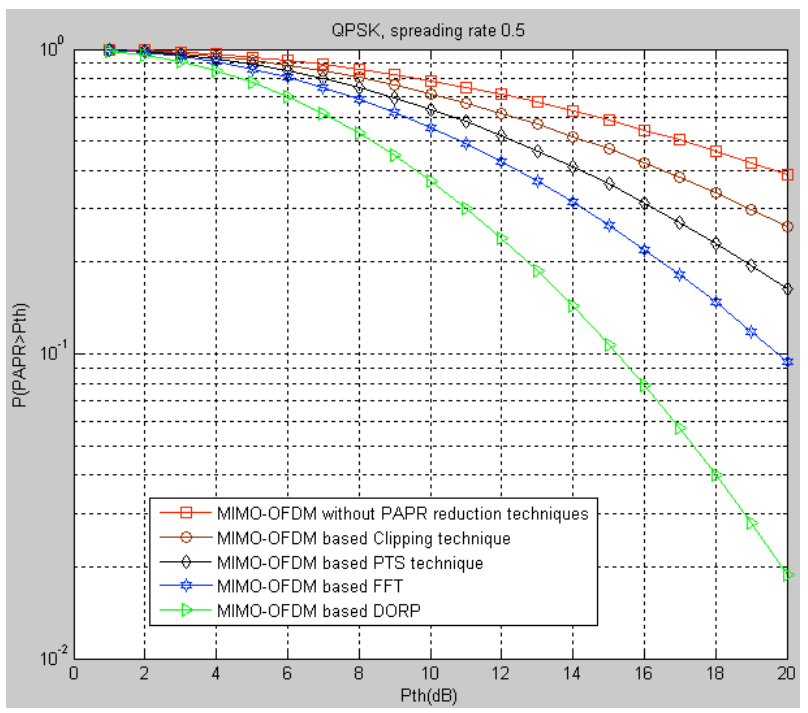

Figure 4. Comparison between the probabilities of PAPR values that exceed a certain threshold for the proposed work comparing to the conventional MIMO-OFDM system (for QPSK modulation process). 


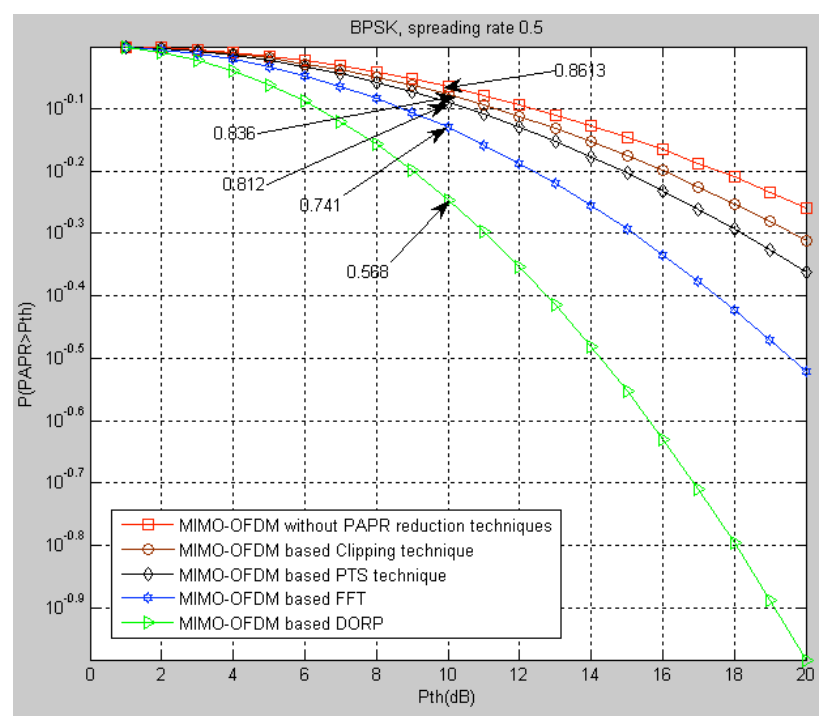

Figure 5. Comparison between the probabilities of PAPR values that exceed a certain threshold for the proposed work comparing to the conventional MIMO-OFDM system (for BPSK modulation process).

duction techniques. At $20 \mathrm{~dB}$ threshold, the CCDF curve for the conventional MIMO-OFDM system shows a reduction from $40 \times 10^{-2}$ to $91 \times 10^{-3}$, while this value has been improved to reach $9 \times 10^{-4}$ after using the proposed algorithm. The same result has been achieved from Figure 5 when the modulation technique has been changed to be BPSK; from this Figure using the proposed work reduces the PAPR value from $55 \times 10^{-2}$ to $10.4 \times 10^{-2}$.

\section{CONCLUSION}

This paper has introduced DORP, which is a new proposition of designing the OFDM transceivers. This proposition is based on identifying and analyzing the peaks and valleys of OFDM signal. This algorithm is based on Denoise the OFDM using some DWT, after that defining an adaptive threshold to limit those peaks, and finally replace these peaks and valleys using an average filter. This algorithm gives an enhancement around $45 \%$ of reducing the peaks production. The analytical derivation of the technique has been given which describes the theoretical functionality of the technique. Simulation results which are consisting of three different parts indicated that the new design could improve the MIMO-OFDM systems performance even in a condensed Multipath channel.

A performance comparison between the proposed work and the used techniques in the literature has been made. From this comparison, the DORP shows a potential and gives a performance improvement between $32 \%-46 \%$ for different modulation techniques.

\section{REFERENCES}

[1] N. Brümmer, "Application-independent evaluation of speaker detection," in Proc. Odyssey: Speaker Lang. Recognition Workshop, 2004, pp. 33-40.

[2] J. Gonzalez-Rodriguez, A. Drygajlo, D. Ramos-Castro, M. GarciaGomar, and J. Ortega-Garcia, "Robust estimation, interpretation and assessment of likelihood ratios in forensic speaker recognition," Comput. Speech Lang., vol. 20, no. 2-3, pp. 331-355, 2006. http://dx.doi.org/10.1016/j.csl.2005.08.005

[3] W. M. Campbell, K. J. Brady, J. P. Campbell, R. Granville, and D. A. Reynolds, "Understanding scores in forensic speaker recognition," in Proc. Odyssey: Speaker Lang. Recognition Workshop, 2006.

[4] Khaizuran Abdullah And Zahir M. Hussain, "Studies On DwtOfdm And Fft-Ofdm Systems",International Conference On Communication, Computer And Power (Icccp'09), Muscat, February 15-18, 2009.

[5] M. Breiling, S. H. Muuller, and J. B. Huber, "SLM peak power reduction without explicit side information," IEEE Commun. Lett., vol. 5, no. 6, pp.239-241, 2001. http://dx.doi.org/10.1109/ 4234.929598

[6] J. Tellado, Multicarrier Modulation with Low PAR. Applications to DSL and Wireless. New York: Kluwer Academic Publishers, 2002.

[7] A. Ben Aicha, F. Tilli, S. Ben Jebara, PAPR analysis and reduction in WPDM systems, in: IEEE first international symposyiom on control, communications and signal processing, 2004, pp. 315318.

[8] Volkan Kumbasar, Oguz Kucur, Better wavelet packet tree structure for PAPR reduction in WOFDM systems, Science Direct, Digital signal processing 18(2008), pp. 885-891. http://dx.doi.org/10.1016/j.dsp.2008.06.003

[9] H. Zhang, D. Yuan, and M. Patzold, "Novel study on PAPRs reduction in wavelet-based multicarrier modulation systems", Science Direct, Digital signal processing 17, pp. 272-279, 2007. http://dx.doi.org/10.1016/j.dsp.2006.08.002

[10] M. Al-Akaidi, O. Daoud, and S. Linfoot, "A new Turbo Coding Approach to reduce the Peak-to-Average Power Ratio of a MultiAntenna-OFDM", International Journal of Mobile Communications, vol. 5, no.3, pp. 357-369, 2007. http://dx.doi.org/10.1504/IJMC.2007.012399

[11] R. C. Gonzales, R. E.Woods, and S. L. Eddins. Digital Image Processing. Prentice Hall, 2008.

[12] Donoho, D., De-noising by Soft-thresholding., IEEE Trans. Information Theory, Vol. 41, No. 3, pp. 613-627, 1995.

\section{AUTHORS}

Omar Daoud is with the Dept. of Communications and Electronics Engineering, Philadelphia University, Amman, Jordan (odaoud@philadelphia.edu.jo)

Qadri Hamarsheh is with the Dept. of Computer Engneering, Philadelphia University, Amman, Jordan.

Wael Al-Sawalmeh is with the Dept. of Communication Engineering, Al-Hussin Bin Talal University, Ma'an, Jordan.

Submitted 07 October 2012. Published as re-submitted by the authors 26 June 2013. 